\title{
A CLASS OF IRREDUCIBLE SYSTEMS OF GENERATORS FOR INFINITE SYMMETRIC GROUPS
}

\author{
R. B. $\mathrm{CROUCH}$
}

If $G$ is a group and $M$ a subset of $G$ then $\{M\}$ is the smallest subgroup of $G$ containing $M$. If $\{M\}=G$ then $M$ is a system of generators for $G$. If no proper subset of $M$ is a system of generators for $G$ then $M$ is irreducible.

Let $N$ be the set of positive integers; $d$ the cardinal number of $N$; $d^{+}$the successor of $d ; S\left(d, d^{+}\right)$the group of all one-to-one mappings of $N$ onto itself; $A(d, d)$ the alternating subgroup of $S\left(d, d^{+}\right) ; S(d, d)$ the finite symmetric subgroup of $S\left(d, d^{+}\right)$.

THEOREM 1. Let $M$ consist of the sequence of odd length cycles of $S\left(d, d^{+}\right)$

$\left(1,2, \cdots, n_{1}\right),\left(n_{1}, n_{1}+1, \cdots, n_{2}\right), \cdots$,

$$
\left(n_{i}, n_{i}+1, \cdots, n_{i+1}\right), \cdots
$$

with the order of the cycles $s_{i}=k_{i} \geqq 3$. Then $M$ is an irreducible system of generators for $A(d, d)$.

Proof. It is clear from the nature of the set $M$ that $\{M\} \subseteq A(d, d)$. Furthermore, if $c_{i}$ is removed from $M$ then every element of the group generated by the remaining set leaves the integer $n_{i-1}+1$ fixed. It is sufficient, therefore, to prove that every element of $A(d, d)$ belongs to $\{M\}$. Since the 3 -cycles generate $A(d, d)$ we shall show any 3 -cycle belongs to $M$.

Let $x_{1}<x_{2}<x_{3}$ be any triple of elements of $N$. There exists an element $s_{i}$ of $M$ such that $x_{i} \in s_{i}$ and $x_{i}$ is not the greatest element of $s_{i}$, $i=1,2,3$. Furthermore, there exists a positive integer $\alpha_{i}$ such that $s_{i}^{\alpha_{i}}\left(x_{i}\right)=m_{i}$ where $m_{i}$ is the largest integer in $s_{i}$. In the set $M$ choose the cycle, say $s_{0}$, which is the immediate successor of $s_{3}$ in the sequence of cycles of $M$. Denote by $s_{i 1}, s_{i 2}, \cdots, s_{i r_{i}}$ the elements of $M$ which occur in the sequence between $s_{i}$ and $s_{0}$. Consider the product

$$
s_{i}^{\alpha_{i}-1} s_{i 1} s_{i 2}^{-1} \cdots s_{i r_{i}}^{-1} s_{0} s_{i r_{i}} \cdots s_{i 2} s_{i 1} s_{i}^{t_{i}-\alpha_{i}}
$$

where $t_{i}$ is the order of $s_{i}$. A computation shows that this product is

$$
\left(x_{1}, a_{2}, \cdots, a_{p}\right)
$$

where $s_{0}=\left(a_{1}, a_{2}, \cdots, a_{p}\right)$. Denote by $d_{1}, d_{2}, d_{3}$ the three cycles that

Presented to the Society, April 18, 1959; received by the editors March 7, 1959. 
the above formula yields. Now compute $d_{1} d_{2}^{-1}$ and $d_{1} d_{3}^{-1}$ which yield $\left(x_{1}, x_{2}, a_{p}\right)$ and $\left(x_{1}, x_{3}, a_{p}\right)$. A final computation of $d_{1} d_{2}^{-1} d_{3} d_{1}^{-1}$ shows that $\left(x_{1}, x_{2}, x_{3}\right)$ belongs to $M$.

THEOREM 2. Let $M$ consist of the sequence of cycles of $S\left(d, d^{+}\right)$, where $c_{1}$ is of even length, $\left(1,2, \cdots, n_{1}\right),\left(n_{1}, n_{1}+1, \cdots, n_{2}\right), \cdots$,

$$
\left(n_{i}, n_{i}+1, \cdots, n_{i+1}\right), \cdots
$$

with the order of the cycles $s_{i}=k_{i} \geqq 4$. Then $M$ is an irreducible system of generators for $S(d, d)$.

Proof. By an argument similar to the one given above, it is clear that $A(d, d) \subseteq M$. If $x_{1}, x_{2}$ are any elements of $N$ and $c_{1}=\left(1,2, \cdots, n_{1}\right)$ then $\left(x_{1}, x_{2}\right) c_{1}$ is a member of $A(d, d)$, hence in $\{M\}$. But $c_{1}$ belongs to $M$, hence to $\{M\}$ and $\left(x_{1}, x_{2}\right) c_{1} c_{1}^{-1}=\left(x_{1}, x_{2}\right)$ is in $\{M\}$.

Corollary. There exists $d^{d}$ irreducible systems of generators for $S(d, d)$ and $A(d, d)$.

Theorem 3. Let $M$ consist of all elements of the form $(i, i+1)$, $i=1,2, \cdots, n, \cdots$. Then $M$ is an irreducible system of generators for $S(d, d)$.

Proof. Let $r<s$ be any distinct elements of $N$. Then the formula $(r, r+1)(r+1, r+2) \cdots(s-1, s)(s-2, s-1) \cdots$

$$
(r+1, r)=(r, s)
$$

shows that $M$ contains any transposition. The set $M$ is irreducible because if $M_{1}$ is $M$ with $(i, i+1)$ removed then $M_{1}$ does not contain $(i+1, x)$ for $x>i+1$.

New Mexico State University 